\title{
WS2-B04
}

\section{Using Inversion to Estimate the Impact of Broadband Data on Elastic Property Uncertainties}

\author{
J.V.R. Townend* (Maersk Oil) \& A.J. Cherrett (Maersk Oil)
}

\section{SUMMARY}

We present a comparison of broadband and conventional data using joint elastic inversion to quantify posterior uncertainties. The uncertainty can be quantified at different scales, for example according to temporal frequency or layer thickness. Our observations confirm that enhancements to the usable bandwidth in extreme high and low seismic frequencies give rise to a quantifiable uplift in resolution across a full range of scale lengths. 


\section{Introduction}

Recent advances in seismic data acquisition and processing methods have provided an increase in seismic bandwidth. This is expected to improve spatial resolution and reduce uncertainty within the fields of seismic reservoir characterisation and elastic inversion.

The example given employs seismic data covering a North Sea clastic oil reservoir that have been recently acquired using a slanted streamer approach (Soubaras, 2010). This is compared to an existing conventionally acquired equivalent dataset. Processing of these new data is still underway, but at an early stage, a migrated mini-cube around a key well has been used to investigate its effect on the uncertainties of properties estimated by elastic inversion. Wavelets at reflection angles of $9.5^{\circ}, 18.5^{\circ}$ and $27.5^{\circ}$ were extracted from both datasets during the well-tie process (Figure 1); those from the slanted streamer data exhibit expected bandwidth enhancements at both low and high frequencies.

\section{Method}

The joint elastic inversion method described by Buland et al. (2003) can be used to compute the posterior mean and covariance for both datasets under quite strict assumptions of stationarity and Gaussianity. In the Fourier domain the posterior covariance diagonalises, so that the covariance can be decomposed by temporal frequency (Figure 2). By convolving the posterior covariogram with geobodies it is possible to compute the variance of the average properties in those bodies. As an example: we use horizontal layers of different time-thicknesses to give posterior standard deviation as a function of time-thickness (Figure 3).
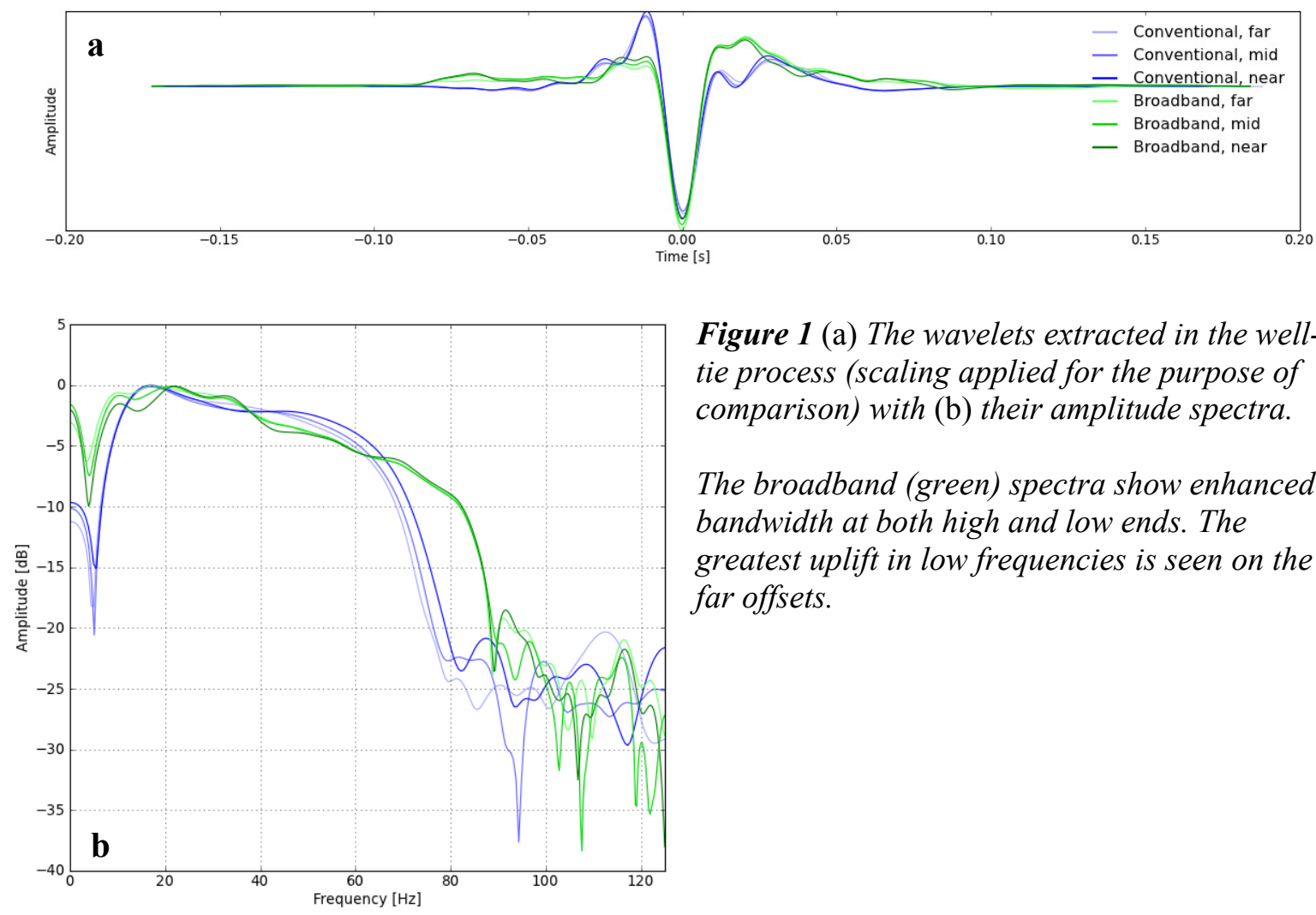

Figure 1 (a) The wavelets extracted in the welltie process (scaling applied for the purpose of comparison) with (b) their amplitude spectra.

The broadband (green) spectra show enhanced bandwidth at both high and low ends. The greatest uplift in low frequencies is seen on the far offsets.

\section{Discussion}

The slanted steamer data exhibit a greater range of usable bandwidth, expanded over the conventional data in both low and high frequencies. When using this broader band data there are reduced uncertainties of properties estimated by elastic inversion across a range of scale lengths. With a current 


\section{Amsterdam $\mid ' 14$}

maximum reflection angle of $27.5^{\circ}$ used in the inversion, the uncertainty in $\mathrm{V}_{\mathrm{P}} / \mathrm{V}_{\mathrm{S}}$ remains relatively high with the acoustic impedance benefiting most from the broadband data.

We expect the final volumes from the new acquisition to contain less noise than the previous data, due to the use of more recent equipment and processing along with a deeper tow depth for much of the streamer length. This example assumes the same signal-to-noise ratio in both datasets, in order to illustrate and isolate the bandwidth-related improvements.
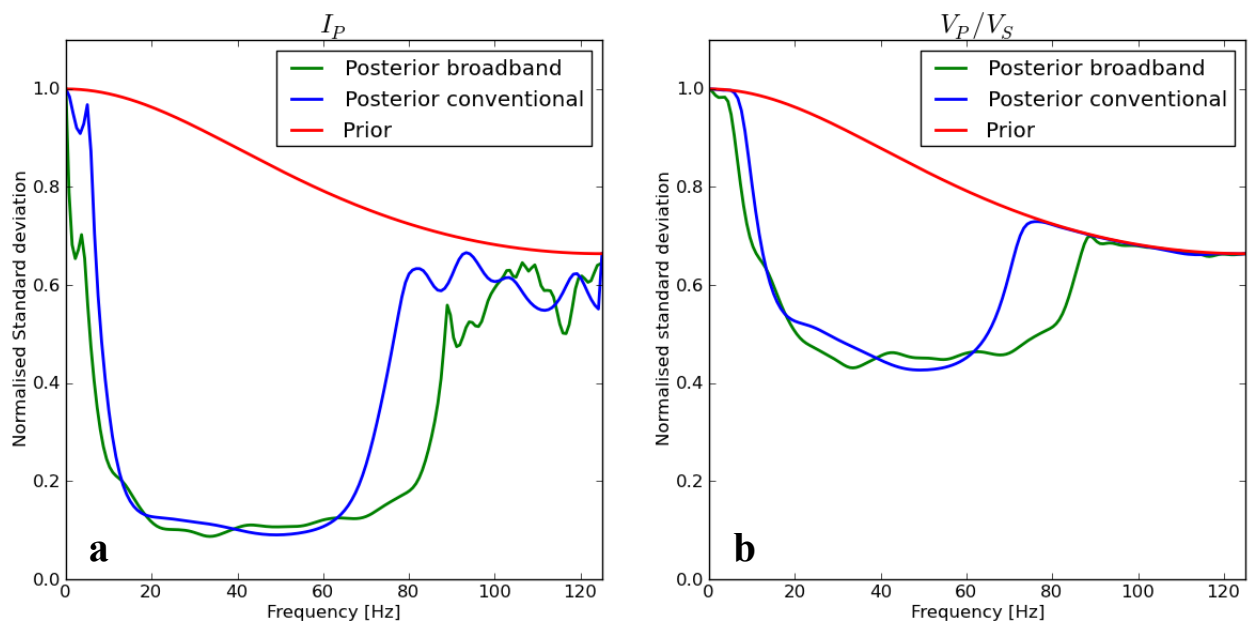

Figure 2 Posterior uncertainty against frequency for (a) acoustic impedance and (b) $V_{P} / V_{S}$.
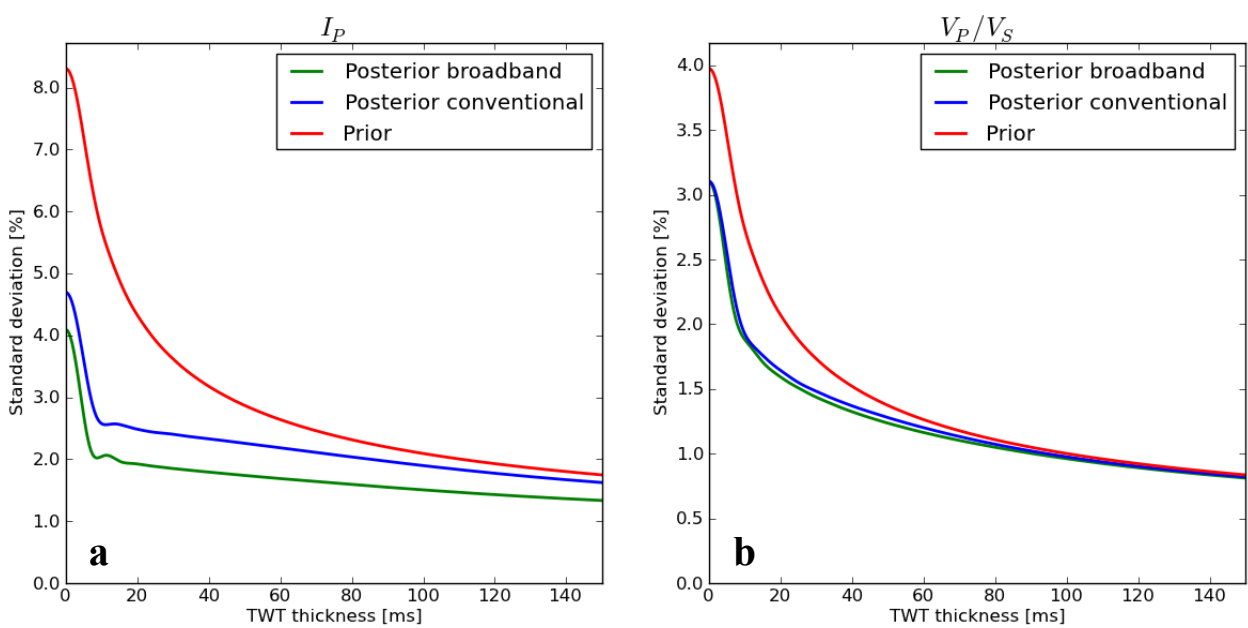

Figure 3 Posterior uncertainty against TWT thickness for (a) acoustic impedance and (b) $V_{P} / V_{S}$.

\section{Acknowledgements}

We would like to thank our partners for permission to use these datasets, Hanno Klemm for help with wavelet extraction as well as the CGG Maersk Oil in-house dedicated processing centre.

\section{References}

Buland, A., Kolbjørnsen, O. \& Omre, H. [2003] Rapid spatially coupled AVO inversion in the Fourier domain. Geophysics, 68, 824-836.

Soubaras, R. [2010] Deghosting by joint deconvolution of a migration and a mirror migration. $80^{\text {th }}$ SEG Annual International Meeting, Expanded Abstracts, 3406. 